\title{
Benign Inverted Papilloma with Intracranial Extension: Prognostic Factors and Outcomes
}

\author{
Ernest J. Wright, B.A., B.S., ${ }^{1}$ Natalya Chernichenko, M.D., ${ }^{2}$ Eylem Ocal, M.D., ${ }^{1}$ \\ Jennifer Moliterno, M.D., 1 Ketan R. Bulsara, M.D., \\ and Benjamin L. Judson, M.D. ${ }^{2,3}$
}

\section{ABSTRACT}

We describe a case of benign inverted papilloma with intracranial extension treated with endoscopic resection combined with craniotomy. Intracranial involvement of inverted papilloma, in the absence of malignancy, is uncommon. We present an analysis of the literature identifying the characteristics and outcomes of benign intracranial inverted papilloma. PubMed database was searched using keywords intracranial, inverted or inverting, and papilloma. There are 17 reports of benign inverted papilloma with intracranial extension reported with a mean age of 49.2 years (range, 23 to 92 years), a female predominance, $22 \%$ of cases with an associated mucocele, and $60 \%$ recurrent disease. The most common sites of invasion are the frontal sinus or cribriform plate. The prognosis for benign intracranial inverted papilloma is dependent on the presence of dural invasion and the achievement of total resection. There are no reported recurrences after craniofacial resection with a mean follow-up of 7.9 years. Adjuvant radiation therapy has demonstrated benefit in cases of residual disease after resection. We expect that endoscopic resection, the standard treatment for sinonasal inverted papilloma, will be increasingly used in the presence of intracranial extension.

KEYWORDS: Intracranial, inverted, inverting, Schneiderian, papilloma

Inverted papilloma is a benign yet locally aggressive neoplasm of the paranasal sinuses with a propensity for recurrence and the potential for malignant transformation. Inverted papilloma with intracranial extension in the absence of malignant transformation represents a rare variant of this tumor, often the result of recurrent disease with a dire prognosis in the event of dural invasion. In this report we present our patient with intracranial inverting papilloma and a comprehensive analysis of all such cases reported in the literature, identifying the characteristics of this rare variant of intracranial inverted papilloma and assessing prognostic factors.

\section{CASE REPORT}

A 32-year-old African male presented to the emergency department complaining of progressive proptosis over the course of 1 year accompanied by increasing difficulty breathing through his left nostril for the past 3 months. The patient denied pain, epistaxis, rhinorrhea, diplopia, or headache. Physical examination revealed a pink, polypoid mass inside the patient's left nostril as well as nonpulsatile proptosis of the left eye. Neurological examination yielded no focal deficits, with visual acuity, pupillary reaction to light, and extraocular movements intact bilaterally.
Departments of ${ }^{1}$ Neurosurgery, ${ }^{2}$ Otolaryngology, and ${ }^{3}$ Surgery, Yale University School of Medicine, New Haven, Connecticut.

Address for correspondence and reprint requests: Benjamin L. Judson, M.D., Assistant Professor, Section of Otolaryngology, Department of Surgery, 800 Howard Avenue, New Haven, CT 06519-1369 (e-mail: benjamin.judson@yale.edu).

Skull Base Rep 2011;1:145-150. Copyright (C) 2011 by Thieme
Medical Publishers, Inc., 333 Seventh Avenue, New York, NY 10001, USA. Tel: +1(212) 584-4662.

Received: June 9, 2011. Accepted: June 17, 2011. Published online: September 29, 2011.

DOI: http://dx.doi.org/10.1055/s-0031-1287687.

ISSN 2157-6971. 
Computed tomography scan revealed an infiltrative mass with opacification of the left maxillary, sphenoid, and frontal sinuses and extension into the left orbit. To better delineate the extent of involvement, magnetic resonance imaging (MRI) was performed demonstrating a large mucocele involving the left maxillary sinus and sphenoid sinus. Additionally, a sinonasal mass extended intraconally into the left orbit and intracranially into the left anterior cranial fossa (Fig. 1). An endoscopic biopsy was performed to establish a diagnosis, revealing inversion of a metaplastic squamous epithelium consistent with inverted papilloma.

Given a presumptive diagnosis of inverted papilloma, the patient underwent an endoscopic resection of the sinonasal portion of the tumor and a bifrontal craniotomy for resection of the lateral intracranial and the intraconal portions of the tumor with the objective of complete resection. Transnasal endoscopic resection was used to remove the intranasal component of the tumor. An endoscopic medial maxillectomy and total ethmoid-sphenoidotomy was performed removing the tumor from these sites. The cribriform plate was intact and free of disease, but tumor was still present in the lateral supraorbital air cell that extended beyond the midline of the orbit and was not well-visualized endoscopically. A bifrontal craniotomy was performed and a large vascularized pericranial graft was obtained. The frontal sinus was cranialized, and the left orbit was exposed from above. The tumor appeared to have arisen in the lateral supraorbital air cell and eroded through the roof of this cell intracranially and through the floor into the orbit. Tumor was removed from the anterior cranial fossa, supraorbital air cell, and intraconal orbit. The bone underlying the tumor stalk was removed with a drill. Gross examination revealed no dural infiltration. The pericranial graft and a titanium plate over the orbit were used to reconstruct the floor of the anterior cranial fossa.
The patient had an uneventful postoperative course without neurological sequelae: The patient denied diplopia, visual acuity was found to be intact, and there was no evidence of cerebrospinal fluid leakage. Histological examination of the tumor specimens revealed extensive squamous metaplasia and focal highgrade dysplasia with no evidence of malignancy or dural invasion. The patient was followed with serial endoscopic examinations and MRIs without evidence of disease at 1 year.

\section{DISCUSSION}

Inverted papilloma is a benign neoplasm originating from the ectodermally derived Schneiderian epithelium lining the nasal passages and paranasal sinuses.

The earliest descriptions of inverted papilloma are found in case reports by Ward (1854) and Billroth (1855). In 1938, Ringertz ${ }^{1}$ described the characteristic histological appearance of a hyperplastic epithelium inverting into the underlying stroma for which this neoplasm is named. The incidence of inverted papilloma is $0.6 / 100,000$ people per year, and it is thought to comprise 0.5 to $4.0 \%$ of primary nasal tumors. ${ }^{2}$ Inverted papilloma most commonly presents in the 5 th to 7 th decades of life ${ }^{3-6}$ and affects males disproportionately with reported male:female ratios ranging from $2: 1$ to $8: 1 .^{3-8}$

Inverted papillomas with intracranial extension in the absence of malignant transformation represent a rare variant of this rare tumor. With only 17 cases of benign inverted papillomas invading the cranial vault reported in the literature, establishing the incidence of this neoplasm is difficult. ${ }^{3-17}$ The best estimation of the frequency of cranial invasion comes from two of the larger case studies of inverted papilloma, with each consisting of over 100 patients and reporting cranial invasion in 1.8 and $2.0 \%$ of cases. ${ }^{6,8}$ Intracranial disease is differentiated

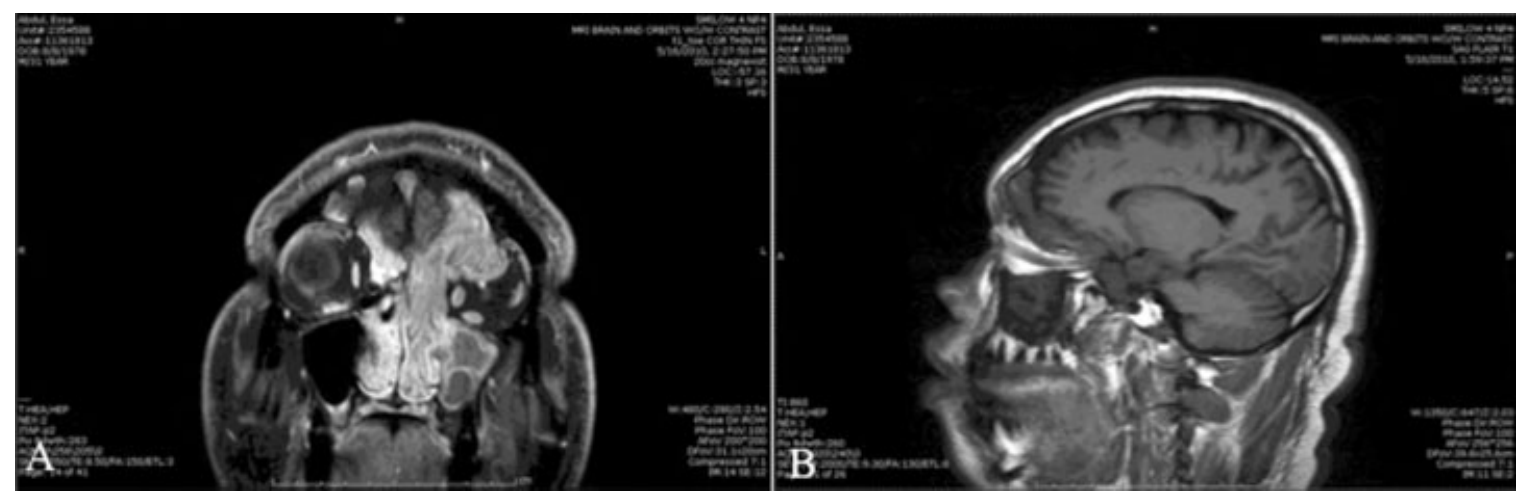

Figure 1 (A) Coronal T1-weighted magnetic resonance image with gadolinium contrast showed an enhancing mass in the left nasal cavity extending into the left frontal sinus and anterior cranial fossa as well as extending into the orbit and compressing the intraorbital structures. The left maxillary sinus shows evidence of postobstructive disease. (B) Sagittal T1-weighted magnetic resonance image demonstrated a mass in the superior left orbit significantly compressing the orbital contents and also extending intracranially. 
from conventional inverted papilloma in that it is often the result of tumor recurrence, with $83 \%$ of the 12 patients in Vural's case series and 60\% of the reported cases in the literature having undergone prior resection (Table 1). Furthermore, intracranial disease is also unique in that it predominantly affects women, with a 1:1.6 male:female ration in the case reports (Table 1). Although it has been suggested that intracranial disease also differs in that it occurs in a younger age group, ${ }^{9}$ a review of the cases reported in the literature does not support this assertion with a mean age of 49.2 years at presentation (Table 1). It has also been proposed that mucocele formation may play an instrumental role in bone necrosis and intracranial invasion, however, less than a quarter $(22 \%)$ of the reports in the literature mention a mucocele as an associated finding (Table 1).

The sites of intracranial extension reported in the literature are listed in Table 1 . The frontal sinus is the most common site of extension ( $47 \%$ of case reports), with the cribriform plate (29\%) and a combination of the frontal sinus and the cribriform plate (18\%) being the next most common. Far less common is extension through the fovea ethmoidalis or the orbit, with only one case of each described in the literature.

The most common presenting symptom of inverted papilloma is unilateral nasal obstruction, followed by epistaxis, nasal discharge, and sinus pressure or pain. ${ }^{3,6-8}$ The symptoms of intracranial disease overlap to a large extent, with the most common being nasal obstruction followed by proptosis and headache (Table 1). With the exception of a single case in which the patient presented with seizures, ${ }^{9}$ there exists no evidence in the literature for reliably distinguishing intracranial disease from conventional inverted papilloma based on symptomatology.

MRI is of utility in determining the extent of tumor invasion and in operative planning, but a definitive diagnosis of inverted papilloma can only be established via biopsy and histological examination demonstrating the characteristic inversion of the hyperplastic epithelium into the stroma with an intact basement membrane, interspersed mucocysts, and varying degrees of cellular atypia. ${ }^{2,7}$

The clinical management of inverted papilloma is dictated by three fundamental characteristics of this neoplasm: its locally aggressive nature, tendency to recur after incomplete resection, and potential for malignant transformation. Inverted papillomas are infiltrative tumors which often present with significant local extension: In two cases series each consisting of over 100 patients, over $95 \%$ of tumors were found to have spread beyond their sites of origin to adjacent sinuses.

Furthermore, inverted papillomas can traverse bony anatomical barriers that would otherwise contain their spread through the destruction of bone by pressure necrosis. Bone erosion and destruction has been observed in 30 to $75 \%$ of cases in various case series. $1,2,18,19$

Inverted papillomas consistently recur after incomplete resection, as evidenced by the substantial difference in recurrence rates seen in limited and radical resection of conventional inverted papilloma. ${ }^{3,6-8,12}$ In the most extensive review of recurrence rates, Myers et al examined 15 published case series of inverted papilloma and found that limited resection yielded recurrence rates between 41 and $78 \%$ in 6/7 studies, whereas radical excision yielded recurrence rates between 0 and $14 \%$ in $8 / 8$ studies. Notably, recurrence can occur years after the initial resection, with Weissler et al reporting 20\% of recurrences occurring at intervals of 5 years or longer postresection.

Inverted papillomas also have a propensity for malignancy. The reported rate of malignant transformation or association with squamous cell carcinoma ranges from 0 to $56 \%$ with the majority of studies reporting rates between 5 and $15 \% .^{3,7,8,20,21}$ Importantly, there have yet to be identified any predictors for malignant transformation: neither cellular atypia, site of origin, nor recurrence have been linked to malignancy. This renders physicians unable to predict the malignant potential of a given lesion. Taken together, the infiltrative nature of inverted papilloma combined with its tendency to recur after incomplete resection and the unpredictable potential for malignancy necessitate aggressive treatment with the objective of complete resection of the tumor.

In cases of inverted papilloma with intracranial extension, craniofacial resection is the best described treatment with good reported outcomes. Assessing the efficacy of craniofacial resection and comparing it to other treatment modalities is difficult because of the limited reports with long-term follow-up. Examining few cases with long-term follow-up data reported in the literature demonstrates that craniofacial resection is highly effective in the majority of patients: $5 / 5$ of patients treated with craniofacial resection for extradural disease were alive at an average follow-up interval of 7.9 years, with only one reported instance of disease recurrence occurring extracranially (Table 1). Craniofacial resection appears to be effective even in cases of intradural disease which portends a very poor prognosis: $2 / 3$ patients treated with craniofacial resection for intradural disease were alive without evidence of disease at follow-up intervals of 3 and 11 years (Table 1). It should be noted that one of the patients with extradural disease and both surviving patients with intradural disease were treated with adjuvant radiotherapy in addition to craniofacial resection.

Transnasal endoscopic resection is the standard treatment for sinonasal inverted papilloma in the more typical situation of the absence of intracranial extension. Transnasal endoscopic approaches are well described with good outcomes for treating both other 


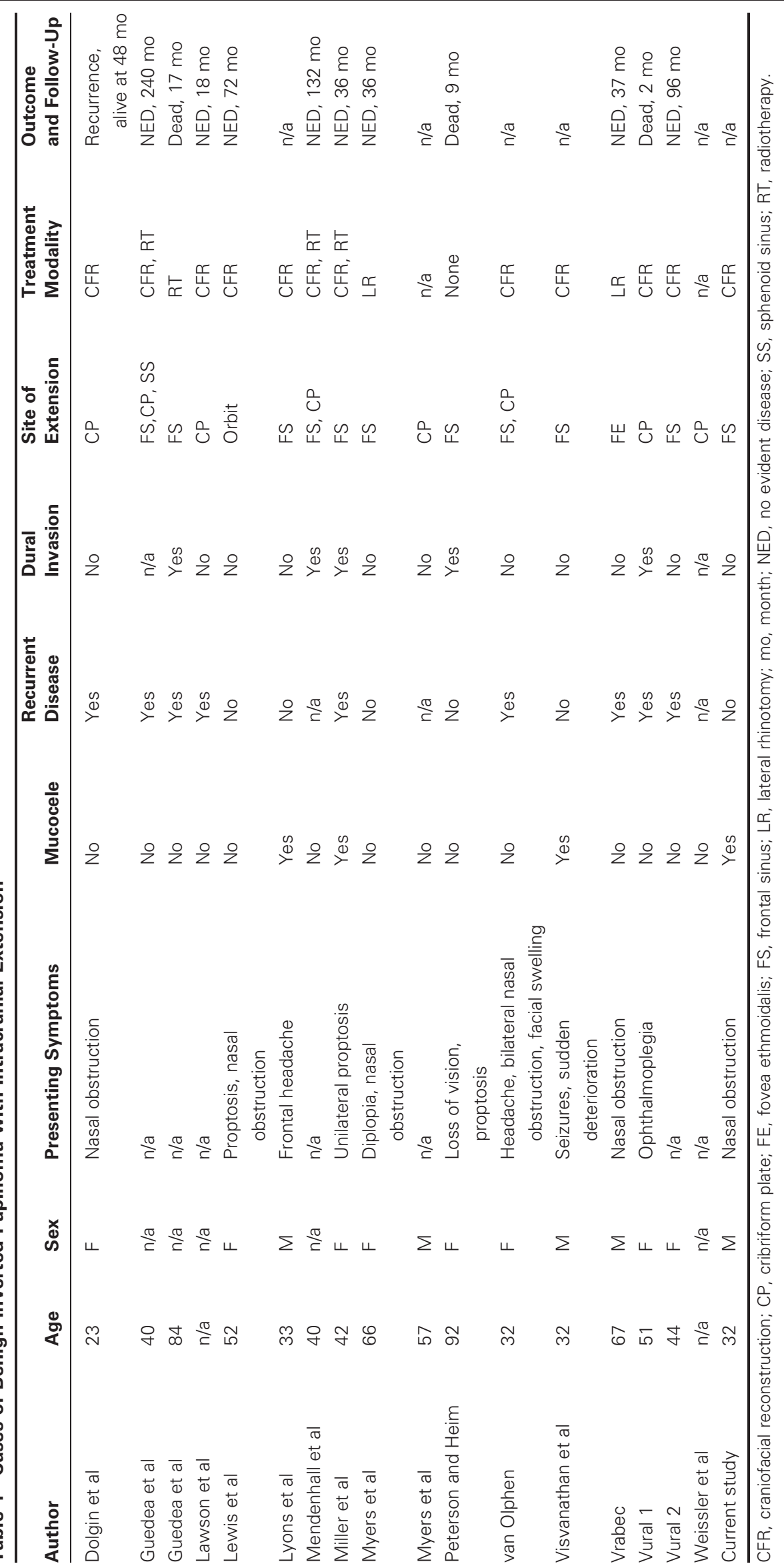


benign as well as selected malignant tumors with intracranial extension. It is likely that the only reason that there are no reported cases of endoscopic management of benign intracranial inverted papilloma with long follow-up is the infrequency of this clinical scenario. We report a case of benign intracranial inverted papilloma treated with endoscopic resection of the sinonasal tumor with a bifrontal craniotomy for removal of lateral intracranial and intraconal portions of the tumor.

In recognition of the fact that residual intracranial disease will almost invariably lead to recurrence and dural invasion, conservative treatment should be avoided if at all possible and reserved only for patients with unresectable lesions or severe comorbid conditions that limit life expectancy or elevate surgical risk to an unacceptable level. The efficacy of radiotherapy as adjuvant treatment for conventional inverted papilloma has been studied and established, ${ }^{16,22}$ but the only data on the efficacy of radiotherapy as primary or adjuvant therapy in intracranial inverted papilloma come from four cases reported in the literature. There are three reports of patients treated with adjuvant radiotherapy (Table 1). ${ }^{15,16,18}$ In two of the reports, the patients had gross residual disease remaining after undergoing craniofacial resection and exhibited no evidence of disease at intervals of 11 and 20 years after radiotherapy. ${ }^{15,16} \mathrm{In}$ a single case the patient was treated with radiotherapy without evidence of residual disease and was diseasefree at 3 years posttreatment. ${ }^{18}$ The fourth case describes the use of radiotherapy as primary treatment for an inoperable lesion, with the patient dying 17 months after treatment. ${ }^{16}$ Taken together, these cases suggest that adjuvant radiotherapy is effective in preventing disease recurrence at intervals of 20 years despite subtotal resection. However, given the high success rates seen with craniofacial resection the use of adjuvant therapy is best reserved for cases with malignant transformation, intradural extension, incomplete resection, or unresectable lesions.

The prognosis for intracranial disease is dependent on dural invasion in addition to achieving total resection. As is the case in conventional inverted papilloma, subtotal resection will almost invariably lead to disease recurrence and poor outcomes. However, in intracranial inverted papilloma, dural invasion is the primary prognostic factor and portends a very poor prognosis in Vural's case series, $75 \%$ of cases of intradural disease had expired at an average follow-up interval of 9 months postoperatively. This is in comparison to a $100 \%$ survival at 4 years for patients with extradural disease for which there was follow-up data.

Long-term follow-up of inverted papilloma with serial endoscopic examination and radiographic imaging is essential given that inverted papillomas can recur at intervals of 5 years or greater posttreatment. ${ }^{7}$

\section{CONCLUSION}

Benign inverted papillomas with cranial invasion represent a unique and rare variant of inverted papilloma, comprising between 1 and $2 \%$ of all cases of inverted papilloma. Intracranial disease is unique in that it is often the result of tumor recurrence that it predominantly affects women, and that prognosis is dependent on dural invasion in addition to the achievement of total resection. Intracranial inverted papilloma displays the fundamental characteristics of inverted papilloma, and consequently complete removal of the tumor mass is required to prevent recurrence, avoid malignant transformation, and achieve good outcomes. Craniofacial resection has demonstrated efficacy in terms of complete removal of intracranial disease and satisfactory follow-up. Endoscopic tumor resection is the standard treatment of inverted papilloma in the absence of intracranial extension and is used to resect other benign and malignant intracranial tumors. It is expected that endoscopic resection will be increasingly used to treat these tumors following the same anatomic guidelines used for resection of other skull base tumors. Our case was resected endoscopically with the addition of a craniotomy to remove intraconal tumor and intracranial tumor lateral to the midline of the orbit. Adjuvant radiation therapy appears to offer some benefit, particularly in complicated or advanced cases. Long-term follow-up for a minimum of 5 years comprising endoscopy and radiographic imaging is essential, given the ability of this tumor to recur at intervals of 5 years or greater after treatment.

\section{REFERENCES}

1. Ringertz N. Pathology of malignant tumors arising in nasal and paranasal cavities and maxilla. Acta Otolaryngol Suppl Stockh 1938;27:31-42

2. Snow JBJR, Wackym PA. Ballenger's Otorhinolaryngology Head and Neck Surgery. 17 ed. Shelton: Pmph USA; 2009

3. Dolgin SR, Zaveri VD, Casiano RR, Maniglia AJ. Different options for treatment of inverting papilloma of the nose and paranasal sinuses: a report of 41 cases. Laryngoscope 1992; 102(3):231-236

4. Myers EN, Schramm VL Jr, Barnes EL Jr. Management of inverted papilloma of the nose and paranasal sinuses. Laryngoscope 1981;91(12):2071-2084

5. Myers EN, Fernau JL, Johnson JT, Tabet JC, Barnes EL. Management of inverted papilloma. Laryngoscope 1990; 100(5):481-490

6. Vrabec DP. The inverted Schneiderian papilloma: a 25-year study. Laryngoscope 1994;104(5 Pt 1):582-605

7. Weissler MC, Montgomery WW, Turner PA, Montgomery SK, Joseph MP. Inverted papilloma. Ann Otol Rhinol Laryngol 1986;95(3 Pt 1):215-221

8. Lawson W, Ho BT, Shaari CM, Biller HF. Inverted papilloma: a report of 112 cases. Laryngoscope 1995;105(3 Pt 1):282-288 
9. Visvanathan V, Wallace H, Chumas P, Makura ZG. An unusual presentation of inverted papilloma: case report and literature review. J Laryngol Otol 2010;124(1): 101-104

10. Vural E, Suen JY, Hanna E. Intracranial extension of inverted papilloma: An unusual and potentially fatal complication. Head Neck 1999;21(8):703-706

11. Mendenhall WM, Million RR, Cassisi NJ, Pierson KK. Biologically aggressive papillomas of the nasal cavity: the role of radiation therapy. Laryngoscope 1985;95(3):344-347

12. Guedea F, Mendenhall WM, Parsons JT, Million RR. The role of radiation therapy in inverted papilloma of the nasal cavity and paranasal sinuses. Int J Radiat Oncol Biol Phys 1991;20(4):777-780

13. Miller PJ, Jacobs J, Roland JT Jr, Cooper J, Mizrachi HH. Intracranial inverting papilloma. Head Neck 1996;18(5):450453; discussion 454

14. Lewis WJ, Richter HA, Jabourian Z. Craniofacial resection for large tumors of the paranasal sinuses. Ear Nose Throat J 1989;68(7):539-547

15. Lyons BM, Donald PJ, Sykes JM, Boggan JE. Craniofacial resection for intracranial inverting papilloma and frontal sinus mucocele. Skull Base Surg 1992;2(2):92-97
16. Peterson IM, Heim C. Inverted squamous papilloma with neuro-ophthalmic features. J Clin Neuroophthalmol 1991; 11(1):35-38

17. van Olphen AF, Lubsen H, van 't Verlaat JW. An inverted papilloma with intracranial extension. J Laryngol Otol 1988; 102(6):534-537

18. Momose KJ, Weber AL, Goodman M, MacMillan AS Jr, Roberson GH. Radiological aspects of inverted papilloma. Radiology 1980;134(1):73-79

19. Phillips PP, Gustafson RO, Facer GW. The clinical behavior of inverting papilloma of the nose and paranasal sinuses: report of 112 cases and review of the literature. Laryngoscope 1990;100(5):463-469

20. Kristensen S, Vorre P, Elbrønd O, Søgaard H. Nasal Schneiderian papillomas: a study of 83 cases. Clin Otolaryngol Allied Sci 1985;10(3):125-134

21. Hyams VJ. Papillomas of the nasal cavity and paranasal sinuses. A clinicopathological study of 315 cases. Ann Otol Rhinol Laryngol 1971;80(2):192-206

22. Hug EB, Wang CC, Montgomery WW, Goodman ML. Management of inverted papilloma of the nasal cavity and paranasal sinuses: importance of radiation therapy. Int J Radiat Oncol Biol Phys 1993;26(1):67-72 